\title{
Comparison of the Analgesic Effects of Ultrasound Guided Supra-Inguinal Fascia Iliaca Block with Femoral Nerve Block for Surgical Fixation of Hip Fractures
}

\author{
Shivani Manohara* ${ }^{\mathbb{C}}$, Yean Chin Lim, Qian Jun Tong \\ Department of Anaesthesia and Surgical Intensive Care, Changi General Hospital, Singapore \\ Email: *manohara.shivani@singhealth.com.sg
}

How to cite this paper: Manohara, S., Lim, Y.C. and Tong, Q.J. (2021) Comparison of the Analgesic Effects of Ultrasound Guided Supra-Inguinal Fascia Iliaca Block with Femoral Nerve Block for Surgical Fixation of Hip Fractures. Open Journal of Anesthesiology, 11, 325-333.

https://doi.org/10.4236/ojanes.2021.1111032

Received: October 7, 2021

Accepted: November 7, 2021

Published: November 10, 2021

Copyright $\odot 2021$ by author(s) and Scientific Research Publishing Inc. This work is licensed under the Creative Commons Attribution International License (CC BY 4.0).

http://creativecommons.org/licenses/by/4.0/

\begin{abstract}
Introduction: Regional anaesthesia techniques have been used for perioperative analgesia for hip fractures. The supra-inguinal approach to fascia iliaca block (FIB) can potentially provide superior analgesia compared to femoral nerve block (FNB) by blocking the obturator and lateral femoral cutaneous nerves. We aimed to evaluate the analgesic effect of single shot FIB and FNB for surgical fixation of hip fractures. Methods: After obtaining ethics approval and written, informed consent, 30 patients undergoing surgical fixation of hip fractures were recruited and randomized into 2 groups. Patients received either a single shot FIB or FNB with $0.5 \%$ Ropivacaine $30 \mathrm{mls}$, and a subarachnoid block. Pain scores were assessed pre-operatively, post-block, in recovery and at 24 hours post-operatively. Time to first analgesic, oxynorm consumption, opioid related side effects and block related complications were assessed at 24 hours. Results: There were no statistically significant difference in post-block pain scores, median (IQR) of $0(0-0)$ versus $0(0-0)$ at rest and $3(2-6)$ versus $5(2-6)$ on positioning for spinal; and 24 hour pain scores were $0(0-0)$ versus $0(0-0)$ at rest and $4(2-5)$ versus $5(2-6)$ on movement for FIB and FNB groups respectively. 5 patients from each group required post-operative opioids, post-operative opioids requirement were similar. Conclusions: Though ultrasound guided supra-inguinal FIB was more consistent in blocking the lateral femoral cutaneous nerve then a femoral nerve block, this did not translate to any difference in terms of pain scores, opioid consumption and side effects.
\end{abstract}

\section{Keywords}

Hip Fracture, Regional Anaesthesia, Analgesia, Femoral Block, Fascia Iliaca Block 


\section{Introduction}

Hip fractures are common in the elderly. Many of these patients are frail and have multiple comorbidities. There is a high morbidity and mortality rate, with the 1st year mortality ranging from $14 \%$ - 58\% [1]. Current anesthesia techniques for surgical fixation of hip fractures include general anesthesia, central neuraxial blocks, with peripheral nerve blocks and opioids as the mainstay for postoperative analgesia. Cochrane review by Guay et al. [2] did not show a difference between general anesthesia and regional anesthesia techniques for adult hip fracture surgery except for deep venous thrombosis in the absence of potent thromboprophylaxis.

A study by Morrison et al. [3] suggested that good analgesia decreased length of hospital stay, enhanced functional recovery and long term functional outcomes. To reduce the side effects of opioids in the elderly patients, regional anesthesia techniques may be utilized to potentiate postoperative analgesia. This may hence enhance their recovery and length of hospital stay.

AAGBI safety guideline for management of proximal femoral fractures recommends that peripheral nerve blockade should always be considered as an adjunct to spinal or general anesthesia, to extend the period of postoperative non-opioid analgesia in view of relatively greater risk of respiratory depression and postoperative confusion with usage of opioids in the elderly [4]. Similarly, the National Hip Fracture Database Anesthesia Sprint Audit of Practice 2014 includes consideration of intraoperative nerve blocks for all patients undergoing hip fracture surgery as one of their standards [5].

Our hypothesis is that the supra-inguinal approach to the FIB will provide better analgesia than the FNB when performed under ultrasound guidance as it can potentially block the lateral femoral cutaneous and obturator nerves as well. We conducted a pilot study to investigate the analgesic effects of the FIB compared to the FNB when performed under ultrasound guidance. Secondary outcomes include dermatomal coverage of the suprainguinal approach to the FIB, 24-hour opioid consumption, opioid-related side effects and length of stay.

\section{Materials and Methods}

After obtaining approval from institutional ethics board (SingHealth CIRB) and written, informed consent from patients, 30 patients undergoing surgical fixation of hip fractures were recruited for assessment between August 2014 and March 2015.

Patients with isolated hip fractures, scheduled for surgical fixation under spinal anesthesia, between ages 45 - 90 years old, American Society of Anesthesiologists (ASA) physical status 1 to 3 were recruited. Patients unable to give consent, with inability to cooperate, patients with regular consumption of strong opioids (morphine, oxycodone) or steroids, allergy to local anesthetics or any drugs included in the study and patients with contraindications to spinal anesthesia such as coagulation disorders/thrombocytopenia and local infection at site 
of injection were excluded.

A sample size of 30 was chosen as a feasible number for this pilot study that could be completed in a reasonable time frame based on the current workload in our institution, bearing in mind that there was a large proportion of patients who had to be excluded from the study as they had dementia, were uncooperative or were on dual anti-platelets/anti-coagulants.

Patients were recruited during the pre-operative assessment in the wards once the operating lists were available. A computer generated block randomization with 1:1 ratio, blocks of 10 was used. The patient and data collector were blinded. The block was performed by a study investigator who was independent from the care of the patient. All investigators were proficient in performing ultrasound-guided regional anesthesia and the technique of FIB and FNB were standardized as described. Each patient received either a single-shot fascia iliaca block or femoral nerve block with $0.5 \%$ ropivacaine $30 \mathrm{mls}$ (150 mg of ropivacaine).

The FIB was performed using a suprainguinal approach described by Hebbard et al. [6]. For the FNB, the injection was performed at the level of the inguinal ligament, before the bifurcation of the artery. The needle approach was from lateral to medial and local anesthetic was deposited circumferentially around the nerve. All blocks were done by one of the 2 investigators.

Resting and dynamic pain scores using the numerical rating scale (NRS) before performance of block was assessed. Block success was tested half an hour after performing the block using loss of sensation to cold. Sensory distribution of the block (femoral, obturator and lateral femoral cutaneous nerve) was mapped and recorded. Post-block pain scores were assessed at 30 minutes.

Patient was then turned lateral, with the operative site non-dependent, for performance of spinal anesthesia. Dynamic pain score was assessed upon positioning for spinal anesthesia. Spinal anesthesia is performed with plain bupivacaine $(2.3$ - $3 \mathrm{mls})$ and fentanyl $10 \mathrm{mcg}$. Intravenous ondansetron $4 \mathrm{mg}$ may be given for anti-emesis.

The patient's pain scores were assessed at 4 instances-pre-block, 30 minutes post block, $1 \mathrm{hr}$ and $24 \mathrm{hrs}$ post-operatively. The patient was assessed at 24 hours post-operatively for time to first analgesic (oxynorm), oxynorm consumption in the first 24 hours, and for opioid related side effects.

The primary outcomes were pain scores (at rest and on movement) at $30 \mathrm{mi}$ nutes after the block, and at 24 hours. Secondary outcomes were opioid consumption, opioid-related side effects (nausea/vomiting, sedation) and length of stay. Pain scores were assessed using the NRS (0 - 10).

Patients were also assessed for early complications related to peripheral nerve blocks such as hematoma, infection and neurological deficits.

\section{Statistics}

Data were analysed using SPSS for Windows (SPSS Inc. U.S.A.). Categorical data are presented as percentage and frequency. Parametric numerical data are presented as mean and standard deviation while non-parametric data are pre- 
sented as median (interquartile range). Categorical outcomes were analysed with the Chi-squared test or Fisher's Exact test. Numerical data were compared among the groups with $t$ test and non-parametric data with the Mann-Whitney $\mathrm{U}$ test. A two-tailed $\mathrm{p}$-value of $<0.05$ was taken as statistically significant.

\section{Results}

All patients recruited were used in analysis; there were no drop-outs/withdrawals/ loss to follow up.

The baseline demographics and pre-operative pain scores were similar in both groups, reported in Table 1. All blocks were successful and blocked the sensory distribution of the femoral nerve. The FIB resulted in a blockade in the distribution of the lateral femoral cutaneous nerve in $73 \%$ of cases compared to only $27 \%$ by FNB $(\mathrm{p}=0.03)$. The distribution of the obturator nerve was blocked in $80 \%$ of FIB and $67 \%$ of FNB ( $\mathrm{p}=0.68$ ).

The post-block pain scores were similar at rest, median (IQR) of $0(0-0)$ versus $0(0-0)$ and slightly different on positioning for spinal $3(2-6)$ versus 5 (26), for patients receiving FIB and FNB respectively, as shown in Table 2. 24-hour pain scores were also similar, $0(0-0)$ versus $0(0-0)$ at rest and $4(2-5)$ versus 5 (2 - 6) on movement for FIB and FNB groups respectively. Majority of patients

Table 1. Characteristics and perioperative data of patients receiving Femoral Nerve Block or Fascia Iliaca Block for surgical fixation of hip fracture.

\begin{tabular}{ccc}
\hline & $\begin{array}{c}\text { Fascia Iliaca Block } \\
(\mathbf{n}=15)\end{array}$ & $\begin{array}{c}\text { Femoral Nerve Block } \\
(\mathbf{n}=15)\end{array}$ \\
\hline Age (years) & $76(9)$ & $72(12)$ \\
Gender & $9(60 \%)$ & $7(47 \%)$ \\
Male & $6(40 \%)$ & $8(53 \%)$ \\
Female & & - \\
ASA status & - & $6(40 \%)$ \\
1 & $7(47 \%)$ & $9(60 \%)$ \\
2 & $8(53 \%)$ & $11(37 \%)$ \\
3 & $14(47 \%)$ & $2(7 \%)$ \\
Regular pre-op analgesia & $1(3 \%)$ & $10(33 \%)$ \\
Paracetamol & $12(40 \%)$ & $1(0-2)$ \\
NSAIDs & & $8(5-10)$ \\
Tramadol & $2(0-3)$ & \\
Pre-block pain scores & $7(6-8)$ & \\
At rest & & \\
On movement & & \\
\hline
\end{tabular}

Results displayed as number of subjects (percentage \%) or median (IQR). 
Table 2. Primary outcomes.

\begin{tabular}{lccc}
\hline $\begin{array}{c}\text { Pain scores } \\
\text { (NRS scores })\end{array}$ & $\begin{array}{c}\text { Fascia Iliaca Block } \\
(\mathbf{N}=15)\end{array}$ & $\begin{array}{c}\text { Femoral Nerve Block } \\
(\mathbf{N}=15)\end{array}$ & p value \\
\hline \multicolumn{1}{c}{ Post Block } & & & \\
- At Rest & $0(0-0)$ & $5(0-0)$ & 0.296 \\
$-\quad$ On positioning for spinal & $3(2-6)$ & & 0.877 \\
At 24 hrs post-block & & $0(0-0)$ & 0.334 \\
- At Rest & $0(0-0)$ & $5(2-6)$ & 0.937 \\
\hline
\end{tabular}

Results displayed as median (IQR).

had their surgeries done successfully under spinal anaesthesia and did not require additional opioids. 1 patient in the FNB group required fentanyl $20 \mathrm{mcg}$ and morphine $6 \mathrm{mg}$ post-op; 1 patient in the FIB group had a failed spinal, and was converted to general anaesthesia requiring $80 \mathrm{mcg}$ fentanyl and $3 \mathrm{mg}$ morphine intravenously intra-operatively. For 1 patient in the FNB group, the spinal anaesthesia wore off and required conversion to general anaesthesia. However, no additional opioids were required perioperatively.

5 patients from each group required post-operative opioids in the first 24 hours. Patients in FIB group required 3 (5.6) $\mathrm{mg}$ of oxynorm versus 2.7 (5.3) $\mathrm{mg}$ in FNB group, $\mathrm{p}=0.87$. There were no block-related or opioid-related complications in both groups. The length of stay was 6.3 (1.8) versus 8.2 (4.5) days in FIB and FNB groups respectively, $\mathrm{p}=0.15$.

\section{Discussion}

The innervation of the hip joint is complex. Nerves that supply the hip joint include branches from femoral, obturator, sciatic and superior gluteal nerves [7]. The incision site for anterior approaches is innervated by the lateral femoral cutaneous nerve [8]. Blockade of the femoral, obturator and lateral cutaneous nerve of the thigh can provide adequate analgesia. The lumbar plexus block is the most reliable method of blocking all three nerves [9]. However, it is a deep block (risk of hematoma) and technically challenging. FNB is commonly performed for analgesia. However, it only blocks one of the many nerves supplying the hip joint. FIB, based on the principle of giving a larger volume of local anesthetic that may spread along the fascia plane to the lateral femoral cutaneous nerve and branches of the obturator is postulated to provide better analgesia than FNB [6].

Newman et al. [10] compared the FIB performed under landmark technique with FNB performed under nerve stimulator guidance and showed that the FNB provides better analgesia. However, most of our regional anesthesia techniques are currently performed under ultrasound guidance and a study by Dolan et al. [11] has shown that the nerves are more reliably blocked when the FIB is per- 
formed under ultrasound guidance as compared to landmark technique. The Newman study does not reflect our current practice and there are no studies performed to date comparing ultrasound-guided FIB and FNB.

We found that the ultrasound guided supra-inguinal approach to FIB was more consistent in blocking the lateral femoral cutaneous nerve then a femoral nerve block with the same volume and concentration of local anaesthetic. However, this did not translate to any statistically significant difference in terms of pain scores, opioid consumption or opioid-related side effects. There was a trend towards shorter length of hospital stay though it did not reach statistical significance.

In recent systematic reviews, it has been established that regional techniques are superior to standard care (involving intravenous and/or oral analgesics) enhancing recovery and reducing incidents of morbidity amongst this elderly population prone to hip fractures [3] [12]. Diakomi et al. [13] have also shown that landmark technique of FIB provides more effective analgesia during positioning for spinal (i.e. pain score on movement) compared with intravenous fentanyl, and it adequately controls postoperative pain, reducing opioid consumption during the first postoperative day. This is also seen in our study with regards to FIB.

We hypothesised that the FIB can provide superior analgesia compared to a femoral nerve block as it also blocks the lateral femoral cutaneous nerve and obturator nerve.

Dolans et al. [11] compared the ultrasound guided conventional infra-inguinal approach with loss of resistance approach to the FIB and found that the medial aspect of thigh was blocked in $95 \%$ of cases vs $60 \%$ and lateral aspect was blocked in $90 \%$ vs $77 \%$ of cases respectively. This suggests that ultrasound guided fascia iliaca block is more effective than the loss of resistance approach.

However, we did not find any discernible difference in outcomes between FIB and FNB. Newman et al. [10] concluded that FNB provided superior analgesia compared with FIB, but he used a landmark technique for the performance of FIB, which could disadvantage the FIB group. In our study where we used ultrasound guidance for both groups, we did not find any statistically significant difference in terms of analgesia.

The FIB has become more popular in recent years for surgeries involving hip and upper femur as the FIB allows for more targeted deposition of LA compared to the 3-in-1 block. It produces more reliable blockade of femoral, lateral femoral cutaneous and obturator nerves. Capadevila et al. [14] showed that the FIB technique provided faster and more consistent simultaneous blockade of lateral femoral cutaneous and femoral nerves but inconsistent blockade of obturator nerve, with both blocks done via anterior approach and landmark techniques. With the use of ultrasound guidance, the FIB has become even more precise and reliable as shown by Hebbard et al. [6]. In addition, previous imaging studies has shown the inconsistent and unpredictable spread of LA with regards to the 3-in-1 block [15] [16] thus accounting for its falling out of favour. 
Some of the limitations of our study are as follows. We wanted to standardize the dose/concentration of LA for both blocks, so as not to confuse onset of analgesia as being dose related rather than block related. In doing so, we gave $30 \mathrm{mls}$ LA (more than usually required) [17] for an ultrasound guided FNB, which may in fact have resulted in some spread to the fascia iliaca compartment. This could have accounted for this lack of difference in primary and secondary outcomes between the 2 blocks in our study. Further investigation with smaller doses or cadaveric dye studies may be required to confirm this theory. Additionally, our sample size of 30 patients is small, and may have resulted in a type II error, and the lack of difference in primary and secondary outcomes in our blocks.

Our study had concluded in March 2015; since then new data has emerged regarding the inability to block the obturator nerve via the FIB approach. Of note, Swenson et al. [18] used magnetic resonance imaging to delineate injectate distribution after performance of ultrasound guided FIB and 3-in-1 blocks, which showed no spread of injectate to the obturator nerve in either group of patients. All patients in both groups described a loss of temperature sensation over the medial thigh without any loss of motor power for hip adduction. The patients in our study also had similar sensory loss post block, which we used as a surrogate for gauging effectiveness of obturator block as was the current practice at the time. However, sensory loss over medial thigh is not a characteristic finding of isolated obturator blocks now. Bouaziz et al. [19] defined successful obturator blocks as those with hip adductor weakness, as only $20 \%$ of patients show sensory changes in the medial thigh. Unfortunately, we had not tested for adductor weakness during our data collection to confirm whether the obturator nerve was blocked; it is possible that it was spared.

That being said, FIB is an easy, simple-to-perform block that does not require advanced skills. When done under ultra-sound guidance, it is a safe technique with a high success rate, associated with an opioid sparing effect intra and post-operatively. In our institution, FIB is commonly used for patients undergoing hip surgery, with improved pain management profiles and overall patient satisfaction scores. Further advantages over FNB include the injection site being further away from the surgical incision site, therefore not interfering with the operative procedure; FIB catheters can be placed without affecting surgical approach or sterility. Being essentially a plane block, FIB confers a better safety profile, with less risk of nerve injury during injection or during continuous infusions via catheters. Added to that, the use of ultrasound, may reduce the risk of unintended puncture of surrounding structures, including deep circumflex iliac artery, inferior epigastric artery, external iliac artery, spermatic cord and hernia contents [6] as previously encountered, though rarely, with landmark technique.

\section{Conclusion}

The ultrasound guided supra-inguinal FIB was more consistent in blocking the lateral femoral cutaneous nerve than a FNB. This difference in sensory coverage 
did not translate to any significant benefit in terms of pain scores, opioid consumption and side effects in our study. The FIB is recommended for fixation of hip fractures as it provides effective analgesia with an improved safety profile. FNB has a similar analgesic profile and is an acceptable alternative. Since then, we have instituted FIB catheter insertion for patients whose surgery would be delayed for medical reasons with excellent analgesic coverage and patient satisfaction scores.

\section{Prior presentation:}

Interim data from this work was presented at the $34^{\text {th }}$ Annual European Society of Regional Anesthesia and Pain Therapy Congress in Ljubljana, Slovenia, 2-5 September 2015.

\section{Funding}

Funding received from Changi General Hospital, Singapore; research grant was used for employment of a research assistant.

\section{Conflicts of Interest}

The authors declare no conflicts of interest.

\section{References}

[1] Schnell, S., Friedman, S.M., Mendelson, D.A., et al. (2010) The 1-Year Mortality of Patients Treated in a Hip Fracture Program for Elders. Geriatric Orthopaedic Surgery \& Rehabilitation, 1, 6-14. https://doi.org/10.1177/2151458510378105

[2] Guay, J., Parker, M.J., Gajendragadkar, P.R., et al. (2016) Anaesthesia for Hip Fracture Surgery in Adults. Cochrane Database of Systematic Reviews, 2, CD000521. https://doi.org/10.1002/14651858.CD000521.pub3

[3] Morrison, R.S., Magaziner, J., McLaughlin, M.A., et al. (2003) The Impact of Post-Operative Pain on Outcomes Following Hip Fracture. Pain, 103, 303-311. https://doi.org/10.1016/S0304-3959(02)00458-X

[4] Griffiths, R., Alper, J., Beckingsale, A., et al. (2012) Management of Proximal Femoral Fractures 2011: Association of Anaesthetists of Great Britain and Ireland. Anaesthesia, 67, 85-98. https://doi.org/10.1111/j.1365-2044.2011.06957.x

[5] Boulton, C., Currie, C., Griffiths, R., et al. (2014) Falls and Fragility Fracture Audit Programme National Hip Fracture Database. Anaesthesia Sprint Audit of Practice (ASAP). https://www.nhfd.co.uk

[6] Hebbard, P., Ivanusic, J. and Sha, S. (2011) Ultrasound-Guided Supra-Inguinal Fascia Iliaca Block: A Cadaveric Evaluation of a Novel Approach. Anaesthesia, 66, 300-305. https://doi.org/10.1111/j.1365-2044.2011.06628.x

[7] Birnbaum, K., Prescher, A., Hessler, S., et al. (1997) The Sensory Innervation of the Hip Joint-An Anatomical Study. Surgical and Radiologic Anatomy, 19, 371-375. https://doi.org/10.1007/BF01628504

[8] Onyemaechi, N., Anyanwu, E., Obikili, E., et al. (2014) Anatomical Basis for Surgical Approaches to the Hip. Annals of Medical and Health Science Research, 4, 487-494. https://doi.org/10.4103/2141-9248.139278

[9] Touray, S.T., de Leeuw, M.A., Zuurmond, W.W., et al. (2008) Psoas Compartment Block for Lower Extremity Surgery: A Meta-Analysis. British Journal of Anaesthe- 
sia, 101, 750-760. https://doi.org/10.1093/bja/aen298

[10] Newman, B., McCarthy, L., Thomas, P.W., et al. (2013) A Comparison of Pre-Operative Nerve Stimulator-Guided Femoral Nerve Block and Fascia Iliaca Compartment Block in Patients with a Femoral Neck Fracture. Anaesthesia, 68, 899-903. https://doi.org/10.1111/anae.12321

[11] Dolan, J., Williams, A., Murney, E., et al. (2008) Ultrasound Guided Fascia Iliaca Block: A Comparison with the Loss of Resistance Technique. Regional Anesthesia \& Pain Medicine, 33, 526-531. https://doi.org/10.1097/00115550-200811000-00004

[12] Abou-Setta, A.M., Beaupre, L.A., Rashiq, S., et al. (2011) Comparative Effectiveness of Pain Management Interventions for Hip Fracture: A Systematic Review. Annals of Internal Medicine, 155, 234-245.

https://doi.org/10.7326/0003-4819-155-4-201108160-00346

[13] Diakomi, M., Papaioannou, M., Mela, A., et al. (2014) Preoperative Fascia Iliaca Compartment Block for Positioning Patients with Hip Fractures for Central Nervous Blockade: A Randomized Trial. Regional Anesthesia \& Pain Medicine, 39, 394-398. https://doi.org/10.1097/AAP.0000000000000133

[14] Capdevila, X., Biboulet, P., Bouregba, M., et al. (1998) Comparison of the Three-inOne and Fascia Iliaca Compartment Blocks in Adults: Clinical and Radiographic Analysis. Anesthesia \& Analgesia, 86, 1039-1044. https://doi.org/10.1213/00000539-199805000-00025

[15] Capdevila, X., Biboulet, P., Morau, D., et al. (2002) Continuous Three-in-One Block for Postoperative Pain after Lower Limb Orthopedic Surgery: Where Do the Catheters Go? Anesthesia \& Analgesia, 94, 1001-1006. https://doi.org/10.1097/00000539-200204000-00042

[16] Marhofer, P., Nasel, C., Sitzwohl, C., et al. (2000) Magnetic Resonance Imaging of the Distribution of Local Anesthetic during the Three-in-One Block. Anesthesia \& Analgesia, 90, 119-124. https://doi.org/10.1097/00000539-200001000-00027

[17] Casati, A., Baciarello, M., Di Cianni, S., et al. (2007) Effects of Ultrasound Guidance on the Minimum Effective Anaesthetic Volume Required to Block the Femoral Nerve. British Journal of Anaesthesia, 98, 823-827. https://doi.org/10.1093/bja/aem100

[18] Swenson, J.D., Davis, J.J., Stream, J.O., et al. (2015) Local Anesthetic Injection Deep to the Fascia Iliaca at the Level of the Inguinal Ligament: The Pattern of Distribution and Effects on the Obturator Nerve. Journal of Clinical Anesthesia, 27, 652-657. https://doi.org/10.1016/j.jclinane.2015.07.001

[19] Bouaziz, H., Vial, F., Jochum, D., et al. (2002) An Evaluation of the Cutaneous Distribution after Obturator Nerve Block. Anesthesia \& Analgesia, 94, 445-449.

https://doi.org/10.1213/00000539-200202000-00041 